\title{
LA INDIGNACIÓN COLECTIVA: EL IMPACTO DEL ESTILO PRESIDENCIAL EN LA APROBACIÓN DE LA GESTIÓN Y EL VOTO
}

Luis Costa y Lucas Klobovs ${ }^{2}$ (Argentina)

\section{Introducción}

De algún modo u otro, la idea de los sentimientos ha estado presente en los análisis desarrollados por las ciencias sociales. En las obras del contractualismo clásico, el objeto de estudio se centraba en el individuo y en el modo en que éste lograba relacionarse con los otros, ${ }^{3}$ y desde allí los sentimientos eran un reflejo de su condición natural. Su individualidad condicionaba los modos de la sociabilidad y entonces la sociedad era un reflejo de aquellos condicionamientos.

Luego de abandonar el espíritu spenceriano $0^{4}$ en el siglo XIX y su obsesión por el individualismo, las ciencias sociales comienzan a desarrollar una concepción en la que el orden social y todas sus partes respondían siempre, en algún sentido, a cuestiones que excedían a los individuos

Sociólogo. Director de Cuentas de IPSOS Mora y Araujo. E-mail: luis. costa@ipsos.com.

2 Politólogo. Director de Proyectos de IPSOS Mora y Araujo. E-mail: lucas. klobovs@ipsos.com.

3 Véase, por ejemplo, las diferentes concepciones del hombre entre Hobbes y Locke y cómo éstas dan forma a las posibles soluciones de la convivencia. Ver Hobbes (1980) y Locke (2007).

4 Para Spencer existía una enorme preocupación por el individuo y su libertad junto con una obsesión porque el Estado moderno no limite su particularidad. 
particulares. Sean estas las relaciones de producción ${ }^{5}$ o la religión, ${ }^{6}$ el individuo particular parecía no ser tanto él, sino alguien que en contexto histórico y social se encontraba siempre determinado por condicionantes generales.

El final del siglo XIX y la primera mitad del siglo XX supieron ser gran escenario de experimentos sobre los sentimientos populares. Los nuevos Estados nacionales y su lucha por la construcción de su unidad requirieron de la creación de identidades inventadas con el objetivo de generar consensos especialmente genéricos. ${ }^{7}$ Religiones seculares con objetivo de reponer a la ya perdida hegemonía religiosa fueron las estrellas hasta de los enfrentamientos entre las naciones. Las explosiones sociales de júbilo ante la inminencia de la amenaza de algún vecino, más que el nacimiento de la guerra en sí, muestran el éxito que tuvo la creación de tradiciones.

El sentimiento que aquí particularmente nos interesa es la indignación colectiva. En momentos específicos, la sociedad logra mostrar de una manera bastante homogénea un nivel de molestia compartido; consideramos que esto posee un atractivo especial para el estudio de la opinión pública. Nuestra mayor inspiración en este asunto proviene de Emilie Durkheim y su concepción de la solidaridad mecánica. ${ }^{8}$ A Durkheim le parece especialmente interesante como tema sociológico el hecho de que, ante ciertos crímenes, la sociedad toda se moleste sobremanera.

Marx dice: "Los hombres hacen su propia historia, pero no la hacen a su libre arbitrio, bajo circunstancias elegidas por ellos mismos, sino bajo aquellas circunstancias con que se encuentran directamente, que existen y les han sido legadas por el pasado." Ver Marx (en línea).

Más allá de la obsesión por indicarlo a Weber como un teórico de la acción, su obra es un gran compendio sobre los condicionamientos religiosos en la historia del proceso de modernización. Sobre esta conceptualización ver Weber (1998).

7 Sobre esto recomendamos ver el trabajo pionero de Mosse (2007).

8 Sobre este concepto ver Durkheim (1994). 
Esa reacción espontánea y sin previo acuerdo entre las partes demuestra la unidad de la cultura y nuestra moral unificada. Ese nivel de indignación ante un crimen es poco específico pero muy abarcador, y logra despertar los corazones de casi todos.

Las sociedades experimentan, por momentos, sensaciones de molestia generalizada a las que en algunas ocasiones se intenta describir como rasgos de tipo racional: "La gente se cansó"; "Se reacciona porque le tocaron el bolsillo". Es decir que a una reacción de tipo social se la interpreta sobre condicionamientos individuales, en tanto todos, al mismo tiempo, se unen porque piensan lo mismo. Pero en términos de Durkheim, lo importante es el efecto, y es este mismo efecto (la indignación) el que demuestra la unidad cultural. Como dice Durkheim: "La unidad del efecto demuestra la unidad de la causa."

Este trabajo no tiene como meta mostrar la unidad cultural del argentino y sus reacciones ante estímulos comunicacionales de un gobierno determinado. Nuestro objetivo es plantear una alternativa de medición que permita un primer acercamiento a la comprensión de algo que suele producirse, y que no tiene que ver con acciones o elementos de gobierno concretos. No siempre las oposiciones a una gestión se dan por no estar de acuerdo con la política económica o por temas de educación. En algunos casos es algo menos específico y tangible. Creemos que se puede rechazar a un gobierno por el solo hecho de que sus dirigentes "caen mal".

En este punto hay mucho por explorar en las ciencias sociales, y nuestro interés es sólo plantear la discusión con algunos ejemplos. Nuestra hipótesis es que parte de la mala imagen de la presidenta Cristina Fernández de Kirchner puede ser comprendida por su capacidad de generar un rechazo emocional en algunas personas. El haber logrado alivianar esta cuestión explica en parte su recuperación en 
la valoración de la gente y en consecuencia en su actual intención de voto. Para nosotros, su situación actual, sin este planteo, queda un tanto oscura. Esto es sólo algo más de luz.

Por último, nos parece importante remarcar que los procesos de indignación no son de alcance demasiado largo. Son estados sociales que quiebran la monotonía del orden social cotidiano, y es por eso mismo que llaman la atención.

\section{Desarrollo}

Habitualmente se intenta explicar por qué un presidente tiene buena imagen ante la opinión pública, por qué es valorado y por qué el electorado, al momento de elegir autoridades, termina inclinándose por un candidato y no por otro. Este artículo intentará analizar los cambios registrados en la opinión pública respecto a la valoración presidencial e intención de voto.

La aprobación de la gestión de Cristina Fernández de Kirchner y su intención de voto medido en las encuestas fueron oscilando. La actual mandataria comenzó su gestión con un índice de aprobación del $67 \%$, cayó de forma abrupta a comienzos de $2008,{ }^{9}$ y a partir de diciembre de 2009 comenzó a recuperarse hasta llegar, en la actualidad, a valores de aprobación superiores al $60 \%$. El siguiente gráfico ilustra la evolución:

9 En el inicio de 2008, el Gobierno Nacional promovió un aumento de las retenciones a ciertas exportaciones agrícolas, abriendo un fuerte conflicto con el sector agrícola. Esto generó movilizaciones y protestas por parte de las organizaciones representantes de los intereses del campo. Paralelamente la opinión pública fue tomando posición en este conflicto de fuerte impacto mediático. 
Gráfico 1. Evolución de la aprobación de la gestión de Cristina Fernández de Kirchner

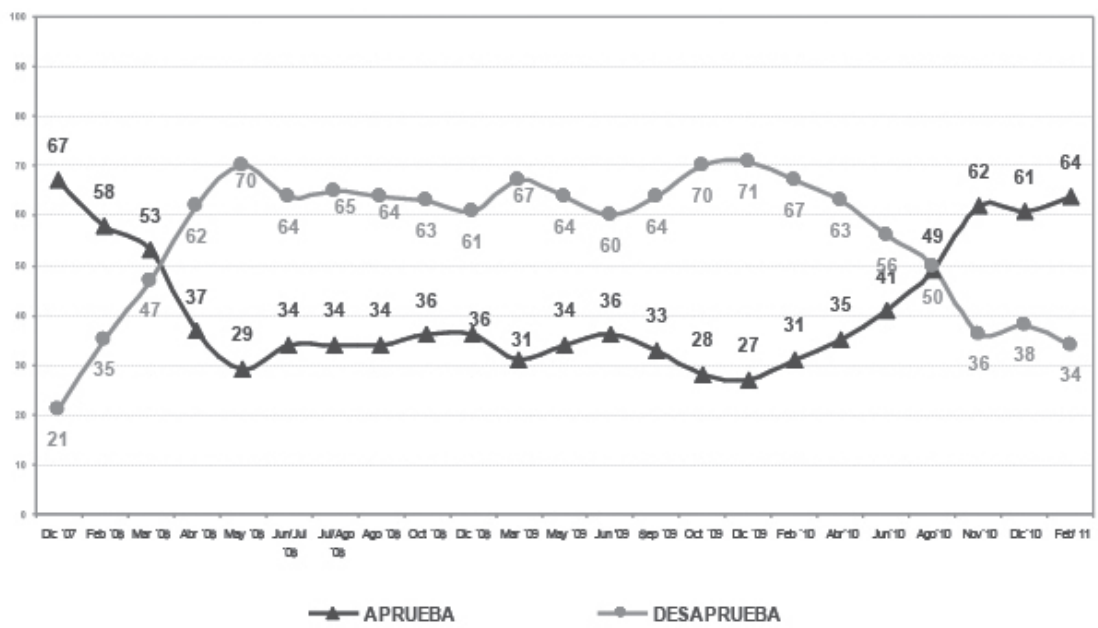

Fuente: IPSOS Mora y Araujo.

Base: 1.200 casos.

De la misma manera, la intención de voto del oficialismo fue en aumento. Si bien es cierto que recién a comienzos del presente año el debate electoral creció, en el año 2010 la intención de voto de Néstor Kirchner y Cristina Fernández de Kirchner no superaba el $20 \%$.

\section{Tipo de motivos de rechazo}

La fuente de información utilizada es la encuesta ómnibus que tiene IPSOS ${ }^{10}$ Argentina. En abril de 2010 realizamos

10 Se trata de una encuesta domiciliaria de 1.200 casos en las principales ciudades del país, probabilística hasta la selección del individuo (luego 
la siguiente pregunta para todos aquellos entrevistados que desaprobaban la gestión de la presidenta Cristina Fernández de Kirchner: “¿Por qué motivos usted desaprueba la gestión de la presidenta Cristina Kirchner?”. Se trató de una pregunta espontánea y de respuesta múltiple.

Los entrevistados señalaron una serie de motivos entre los que se destacaron "no gestiona" (17\%), "es soberbia" (16\%) y "por la inseguridad" (15\%). Si se profundiza en el análisis con el resto de las menciones, pueden detectarse dos clases de motivos: motivos de gestión y motivos personales.

Los primeros hacen hincapié en críticas a la gestión, mientras que los segundos son críticos hacia la persona misma de Cristina Fernández de Kirchner. Es decir, habiendo preguntado por motivos de desaprobación de la gestión, nos encontramos con un importante porcentaje de entrevistados que señalaron causas personales. Este primer análisis nos encamina a cuestionarnos si un porcentaje de la población la desaprueba simplemente por motivos que hacen a sus características personales (estilo, modos o formas) y no por cuestiones de política o de gobierno. En abril de 2010, la distribución de motivos personales y de gestión era la siguiente:

se aplicaron cuotas de sexo, edad y nivel socioeconómico). Se entrevistaron hombres y mujeres de 18 años. La base de datos fue ponderada para respetar los pesos poblacionales de cada zona. 


\section{Gráfico 2. Motivos de desaprobación} de la gestión (abril de 2010)

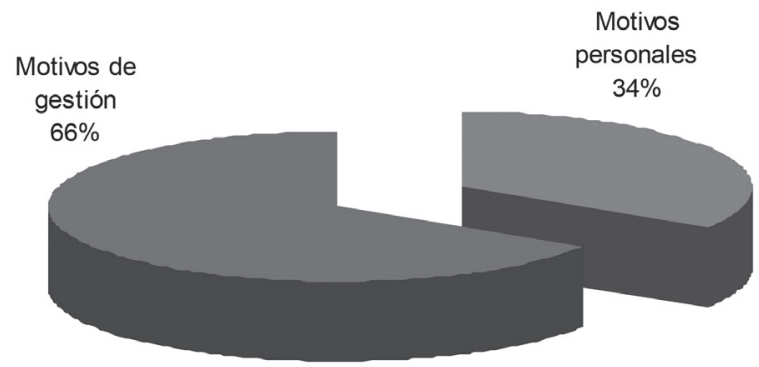

Fuente: IPSOS Mora y Araujo.

Base: 1.200 casos.

\section{Metodología}

Para analizar la desaprobación de Cristina Fernández de Kirchner, agregamos una serie de frases por las cuales el entrevistado debía manifestar su grado de acuerdo o desacuerdo, en una escala de muy de acuerdo, de acuerdo, en desacuerdo y muy en desacuerdo. ${ }^{11}$

Las frases incluidas fueron las siguientes:

1. Cristina Kirchner debería dialogar más con la oposición.

11 La pregunta era la siguiente: "A continuación le voy a leer una serie de frases referidas a la gestión de la presidenta Cristina Kirchner. Para cada una de ellas quisiera que me diga si está muy de acuerdo, de acuerdo, en desacuerdo o muy en desacuerdo." 
2. Me molestan más las formas que tiene el gobierno que las políticas que implementa.

3. El Gobierno Nacional trata a parte de la sociedad despectivamente.

4. Me gustaría que Cristina fuera menos soberbia.

Se le asignó un puntaje a los entrevistados según las respuestas otorgadas, con el siguiente criterio: a mayor puntaje, mayor es el rechazo a los modos que tiene Cristina Fernández de Kirchner. Por lo tanto, los puntos asignados fueron los siguientes:

1. Muy de acuerdo $=4$ puntos.

2. De acuerdo $=3$ puntos.

3. En desacuerdo $=2$ puntos.

4. Muy en desacuerdo $=1$ punto.

Seguidamente se sumaron los puntajes de todos los entrevistados en las cuatro frases analizadas. La sumatoria de los puntajes es una medida resumen del comportamiento del entrevistado. Es decir, la clave es el valor obtenido en las respuestas y no la respuesta a cada frase. Por lo tanto, el entrevistado cuyo puntaje obtenido sea 12 puede haber estado "de acuerdo" en todas las frases (4 x 3), o "de acuerdo" con dos frases, "en desacuerdo" con una y "muy de acuerdo" con otra.

El criterio empleado para determinar los puntajes de corte fue el siguiente: dividir el puntaje obtenido por cada entrevistado por el total de las frases empleadas (4). El cociente que incluía el decimal .50 era el punto de corte (es decir, 1.5, 2.5, 3.5 y 4.5). Si el resultado de la división era 2.5 significa que el entrevistado en promedio respondió 3 (número redondeado, equivalente a la categoría "de acuerdo") a todas las frases. Así se construyeron los siguientes grupos: 
Gráfico 3. Grupos actitudinales (abril de 2010)

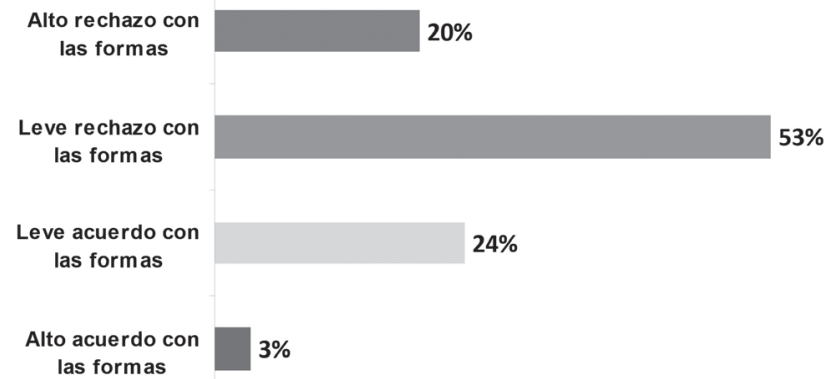

Fuente: IPSOS Mora y Araujo.

Base: 1.200 casos.

La pregunta siguiente busca conocer cómo se posiciona cada grupo según el gobierno de Cristina Fernández de Kirchner y las elecciones a Presidente de octubre del presente año. Si analizamos la aprobación de la gestión vemos lo siguiente: ${ }^{12}$

Tabla 1. Análisis de la aprobación de gestión (abril de 2010)

\begin{tabular}{|c|c|c|c|c|c|}
\hline & Total & $\begin{array}{c}\text { Alto acuerdo } \\
3 \%\end{array}$ & $\begin{array}{c}\text { Leve acuerdo } \\
24 \%\end{array}$ & $\begin{array}{c}\text { Leve rechazo } \\
53 \%\end{array}$ & $\begin{array}{c}\text { Alto rechazo } \\
20 \%\end{array}$ \\
\hline Aprueba & $34 \%$ & $59 \%$ & $61 \%$ & $31 \%$ & $5 \%$ \\
\hline Desaprueba & $64 \%$ & $34 \%$ & $37 \%$ & $68 \%$ & $93 \%$ \\
\hline
\end{tabular}

Fuente: IPSOS Mora y Araujo

Base: 1200 casos

Claramente, se observa que a medida que crece el rechazo a los modos y al estilo que tiene Cristina, la desaprobación crece hasta el 93\%. Incluso es interesante analizar

12 Los porcentajes no suman $100 \%$ porque no se cita el valor del Ns / Nr. 
que entre quienes pertenecen al grupo "alto acuerdo con las formas", hay un $34 \%$ que desaprueba la gestión de la Presidenta. En cuanto a los escenarios electorales ${ }^{13}$ en segunda vuelta, ${ }^{14}$ se daba una situación similar: ${ }^{15}$

\section{Tabla 2: Análisis de los escenarios a segunda vuelta (abril 2010)}

\begin{tabular}{|c|c|c|c|c|c|}
\hline & Total & $\begin{array}{c}\text { Alto acuerdo } \\
3 \%\end{array}$ & $\begin{array}{c}\text { Leve acuerdo } \\
24 \%\end{array}$ & $\begin{array}{c}\text { Leve rechazo } \\
53 \%\end{array}$ & $\begin{array}{c}\text { Alto rechazo } \\
20 \%\end{array}$ \\
\hline Néstor Kirchner & $33 \%$ & $63 \%$ & $56 \%$ & $30 \%$ & $4 \%$ \\
\hline Julio Cobos & $44 \%$ & $10 \%$ & $22 \%$ & $46 \%$ & $74 \%$ \\
\hline Néstor Kirchner & $35 \%$ & $60 \%$ & $56 \%$ & $34 \%$ & $7 \%$ \\
\hline Mauricio Macri & $38 \%$ & $14 \%$ & $22 \%$ & $40 \%$ & $58 \%$ \\
\hline Néstor Kirchner & $36 \%$ & $60 \%$ & $59 \%$ & $34 \%$ & $8 \%$ \\
\hline Elisa Carrió & $35 \%$ & $14 \%$ & $16 \%$ & $38 \%$ & $55 \%$ \\
\hline
\end{tabular}

Fuente: IPSOS Mora y Araujo.

Base: 1.200 casos.

Se observa el mismo fenómeno que con la aprobación presidencial, con el detalle de que Cobos es el dirigente que mayor rédito obtenía en los segmentos que rechazaban los modos de la Presidente. Posiblemente, él representaba el deseo de institucionalidad o pacificación que la sociedad reclamaba en aquel entonces.

El segmento "leve rechazo" que representaba el porcentaje más alto de la población (53\%) se inclinaba por

13 En aquel entonces se midió a Néstor Kirchner como posible candidato a Presidente por el oficialismo.

14 En Argentina, para que haya ballottage el candidato que triunfa en primera vuelta no debe superar el $45 \%$ o llegar al $40 \%$ con una distancia mayor a $10 \%$ respecto al segundo.

15 Los porcentajes no suman $100 \%$ porque no se cita el valor de voto en blanco, voto anulado, entrevistados que señalan que no votarían y los indecisos. 
Cobos (16 puntos por encima del ex Presidente). Este grupo era fundamental en aquel entonces para explicar el caudal electoral del Vicepresidente.

\section{Evolución de los grupos conformados}

Este mismo análisis se realizó en junio, octubre y diciembre con las siguientes salvedades:

1. Se agregaron cinco frases nuevas. Por lo tanto, en lugar de haber cuatro, pasaron a ser nueve las frases analizadas. Por otro lado, se mantuvo el mismo criterio para establecer los puntos de corte entre un grupo y otro.

2. La frase "Cristina Kirchner debería dialogar más con la oposición" fue modificada de la siguiente manera: "Cristina Kirchner dialoga lo suficiente con la oposición". Habiendo aclarado estas cuestiones, se procedió a conformar los grupos:

Gráfico 4. Evolución de los grupos actitudinales

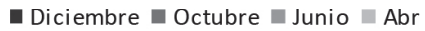

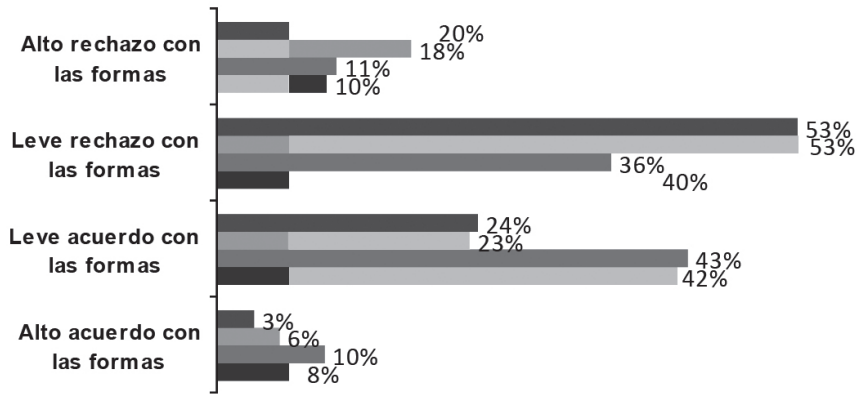

Fuente: IPSOS Mora y Araujo

Base: 1200 casos 
El primer comentario para hacer es que, si bien en abril se utilizaron 4 frases y en junio 9, el comportamiento fue prácticamente el mismo. Por otro lado, la evolución de la conformación de los grupos fue modificándose con claridad. El rechazo al estilo, los modos y las formas de Cristina Kirchner fue disminuyendo. En abril y en junio el alto rechazo ascendía a 20 y $18 \%$ respectivamente, en octubre disminuyó al 11\% y en diciembre se mantuvo en el 10\%. Comportamiento similar obtuvieron los restantes tres grupos.

Si se comparan los datos de abril con diciembre, nos encontramos con que no sólo el peso de cada grupo varió, sino también las opiniones se modificaron:

Tabla 3. Datos comparados en el tiempo

Abril

\begin{tabular}{|c|c|c|c|c|c|}
\hline & Total & $\begin{array}{c}\text { Alto acuerdo } \\
3 \%\end{array}$ & $\begin{array}{c}\text { Leve acuerdo } \\
24 \%\end{array}$ & $\begin{array}{c}\text { Leve rechazo } \\
53 \%\end{array}$ & $\begin{array}{c}\text { Alto rechazo } \\
20 \%\end{array}$ \\
\hline Aprueba & $34 \%$ & $59 \%$ & $61 \%$ & $31 \%$ & $5 \%$ \\
\hline Desaprueba & $64 \%$ & $34 \%$ & $37 \%$ & $68 \%$ & $93 \%$ \\
\hline
\end{tabular}

Diciembre

\begin{tabular}{|c|c|c|c|c|c|}
\hline & Total & $\begin{array}{c}\text { Alto acuerdo } \\
8 \%\end{array}$ & $\begin{array}{c}\text { Leve acuerdo } \\
42 \%\end{array}$ & $\begin{array}{c}\text { Leve rechazo } \\
40 \%\end{array}$ & $\begin{array}{c}\text { Alto rechazo } \\
10 \%\end{array}$ \\
\hline Aprueba & $61 \%$ & $86 \%$ & $85 \%$ & $42 \%$ & $15 \%$ \\
\hline Desaprueba & $37 \%$ & $10 \%$ & $13 \%$ & $57 \%$ & $84 \%$ \\
\hline
\end{tabular}

Fuente: IPSOS Mora y Araujo.

Base: 1.200 casos.

La comparación de estas dos tablas nos lleva a las siguientes conclusiones:

1. La dimensión de los grupos "leve acuerdo" y "leve rechazo" tenían en diciembre el mismo peso, mientras que en abril la situación era diferente. Por lo tanto, a 
principios de 2010, la opinión de este grupo podía llegar a ser determinante.

2. En diciembre, las opiniones de los grupos extremos son marcadamente más opuestas (en abril, el 34\% de quienes formaban el grupo "alto acuerdo" aprobaba la gestión presidencial).

3. Por último, además de que el segmento "leve acuerdo" llega casi a duplicar su dimensión, sus opiniones son marcadamente más kirchneristas.

El segundo análisis implica observar la intención de voto en una hipotética segunda vuelta: ${ }^{16}$

Tabla 4. Datos comparados en el tiempo Abril17

\begin{tabular}{|c|c|c|c|c|c|}
\hline & Total & $\begin{array}{c}\text { Alto acuerdo } \\
3 \%\end{array}$ & $\begin{array}{c}\text { Leve acuerdo } \\
24 \%\end{array}$ & $\begin{array}{c}\text { Leve rechazo } \\
53 \%\end{array}$ & $\begin{array}{c}\text { Alto rechazo } \\
20 \%\end{array}$ \\
\hline $\begin{array}{c}\text { Néstor } \\
\text { Kirchner }\end{array}$ & $33 \%$ & $63 \%$ & $56 \%$ & $30 \%$ & $4 \%$ \\
\hline Julio Cobos & $44 \%$ & $10 \%$ & $22 \%$ & $46 \%$ & $74 \%$ \\
\hline
\end{tabular}

Diciembre

\begin{tabular}{|c|c|c|c|c|c|}
\hline & Total & $\begin{array}{c}\text { Alto acuerdo } \\
8 \%\end{array}$ & $\begin{array}{c}\text { Leve acuerdo } \\
42 \%\end{array}$ & $\begin{array}{c}\text { Leve rechazo } \\
40 \%\end{array}$ & $\begin{array}{c}\text { Alto rechazo } \\
10 \%\end{array}$ \\
\hline $\begin{array}{c}\text { Cristina } \\
\text { Kirchner }\end{array}$ & $57 \%$ & $84 \%$ & $80 \%$ & $39 \%$ & $12 \%$ \\
\hline Julio Cobos & $25 \%$ & $2 \%$ & $10 \%$ & $35 \%$ & $65 \%$ \\
\hline
\end{tabular}

Fuente: IPSOS Mora y Araujo.

Base: 1.200 casos.

16 Dado que en abril el dirigente más favorecido era Julio Cobos, sólo se analizará el ballottage con el Vicepresidente

17 Mientras en abril el candidato oficialista medido fue el ex Presidente, en diciembre fue Cristina Kirchner. 
En cuanto al voto en un hipotético escenario a segunda vuelta, se observa que el grupo más grande vota claramente por la actual Presidente (80\%), mientras que el segundo grupo no se inclina por la oposición como sucedía en abril de 2010.

\section{Conclusión}

Durante el año 2010 se observó un corrimiento en los grupos construidos. Las dos grandes modificaciones fueron las siguientes:

1. Disminución de los grupos que manifestaban rechazo a las formas.

2. Incremento del grupo denominado "leve acuerdo".

Junto con estas nuevas configuraciones, las opiniones tuvieron un comportamiento similar al mencionado con anterioridad.

Los modos y las formas de Cristina Fernández de Kirchner, tal cual son percibidas por la opinión pública, tienen al parecer una incidencia en la construcción de su imagen y en la intención de voto. La percepción de un aparente estilo diferente, junto con otras variables que no están analizadas en este trabajo (como la mejora en la percepción de algunas áreas ${ }^{18}$ ), han influido en la recuperación de la imagen presidencial y su mejor desempeño electoral tal cual se encuentra medido por lo menos en nuestras encuestas.

Según nuestra perspectiva, es bastante poco probable que hoy pueda movilizarse la gente del modo que lo hizo en el pico máximo de tensión con algunos grupos sociales 
del mundo agropecuario. ${ }^{19}$ No sólo podría decirse que el contexto económico es ahora mejor que en la primera mitad de 2008, sino también que al presente le falta algo más intangible: esa indignación.

La sociedad no necesariamente cambia por estos episodios, sino que en todo caso refuerza la idea de que hay algunas cosas que no le gustan. Cuando ese estímulo desaparece, la indignación también se esfuma.

\section{Bibliografía}

Durkheim, Emile (1994), La división del trabajo social, Barcelona, Planeta Agostini.

Hobbes, Thomas (1980), Leviatán, o la materia, forma y poder de una república eclesiástica y civil, Buenos Aires, Fondo de Cultura Económica.

Locke, John (2007), Segundo ensayo sobre el gobierno civil, Buenos Aires, Losada.

Marx Karl (en línea), El 18 brumario de Luis Napoleón Bonaparte. Disponible en línea: http://www.marxists. org/espanol/m-e/1850s/brumaire/brum1.htm.

Mosse, George L. (2007), La nacionalización de las masas, Buenos Aires, Siglo XXI editores.

Weber, Max (1998), Ensayos sobre sociología de la religión I, Madrid, Taurus.

19 Según el diario La Nación del día 15 de julio de 2008, concurrieron a un acto en contra del Gobierno Nacional de la República Argentina unas 237.000 personas. Disponible en línea: http://www.lanacion.com.ar/1030372masivo-apoyo-al-campo-en-el-monumento-de-los-espanoles. 
